\title{
Quantum Computing and the AEC Industry: Trends, Challenges, Near Term Practical Opportunities and Future Outlook
}

This paper was downloaded from TechRxiv (https://www.techrxiv.org).

\section{LICENSE}

CC BY-NC-SA 4.0

SUBMISSION DATE / POSTED DATE

$15-12-2020 / 17-12-2020$

\section{CITATION}

Barima, Oliver (2020): Quantum Computing and the AEC Industry: Trends, Challenges, Near Term Practical Opportunities and Future Outlook. TechRxiv. Preprint. https://doi.org/10.36227/techrxiv.13377674.v1

$\mathrm{DOI}$ 


\title{
Quantum Computing and the AEC Industry: Trends, Challenges, Near Term Practical Opportunities and Future Outlook
}

\author{
Oliver Barima ${ }^{1}$
}

\begin{abstract}
Digital computing and allied communication tools have been phenomenal, but they are fast reaching their technical limits and also their capacity to solve certain extant complex problems. Quantum computing has now emerged with a touted potential to deal with aspects of the latter complex problems and also significantly transform extant computing and communication methods. Yet, little studies exist in the AEC industry related literature on quantum computing to boost awareness and also explore its prospective impact on the AEC industry.

This paper discusses the underpinning basic concepts, technologies and trends in the quantum computing domain to build awareness to boost Architecture, Engineering and Construction (AEC) industry capacity on the topic. The paper dissects the challenges in this novel area. And also examines the near term practical opportunities and potential impact of quantum computing on the AEC industry. The paper concludes with suggestions for practice and future research.
\end{abstract}

Key Words: Quantum computing; AEC Industry; Value delivery; Technology benefits; Technology challenges; Opportunities in Technology use; Computing security risks; Optimization in value delivery; Entanglement ; Superposition

\section{INTRODUCTION}

Computing and allied modern Information and Communication (ICT) tools have so far made significant impacts on society including the Architecture, Engineering and Construction (AEC) industry. Yet, as research and practice seek solutions to deal with complex contemporary and future aspirational needs, the capacity of certain key extant computing and allied ICT tools are fast reaching their technical limits[1,2]. For example, the computational power of conventional computers (including supercomputers) is limited in terms of the problems they can practically solve within specific practical time frames [1,2]. And the use of extant conventional computing techniques will likely require at least hundreds to thousands of years to solve certain extant complex problems in business, science, architecture, engineering and construction value delivery[e.g. $3]$.

Additionally, despite the positive impacts of conventional computing and allied ICT tools, issues such as communication security has been one of the significant challenges in the use of such tools[4]. Security breaches have been common for even powerful governmental and financial institutions with utmost fiduciary duty to preserve the confidentiality of citizens and customers[4]. And over the years identity and other information stolen by criminals have caused enormous consternation for many victims[e.g. 4].

The major drawbacks of conventional computing and allied tools demand fresh ways in dealing with these problems. And this need requires pushing the boundaries of extant knowledge with insights, fresh tools, and transformational approaches to effectively deal with these complex problems. Quantum computing (QC) is one fresh emerging area in computing that is touted to deal with aspects of the latter goal $[1,2,5,6]$. And across industries quantum computing has recently generated a lot of interest among ${ }^{1}$ scholars and practitioners in

\footnotetext{
${ }^{1}$ GB-College, Toronto, Canada. Email:obarima7@gmail.com
} 
search of finding new ways to deal with extant complex problems which conventional computing tools have failed or struggled to solve $[1,2,5,6]$. Little attention, however, has been given to this topical issue in the Architecture, Engineering and Construction (AEC) and allied industries.

Traditionally, the AEC industry plays a key role in providing infrastructure required for the delivery of economic goods and services in many economies. And the AEC industry and allied industries will provide infrastructure and key services that will facilitate the deployment of quantum computing and communication tools. Given the significant aspirational and real impact that quantum computing is likely to play in the global economy over time, it is imperative for the AEC and allied industries to build awareness and also explore potential scholarly and practical implications and challenges that can flow from the probable quantum computing paradigm.

Additionally, the AEC industry can potentially leverage advances in quantum computing to reexamine and deal with persistent complex outstanding problems that classical tools have so far failed to adequately solve. For years AEC industry based research and practice have confronted significant challenges in dealing with very complex issues such as finding optimal value delivery solutions (in for example mega-construction projects)[e.g. 4,7]. Value delivery is an essential element in project delivery, but several projects and constructed products still continue to fail after huge capital and other resource investments[e.g. 7, 8]. This highlights the high costs associated with low value delivery performance [7]. Quantum computing can be a fresh frontier that can help deal with aspects of these challenges through its promise of enormous computing capacity to solve some of the difficult challenges in practical time frames through efficient use of practical resources [e.g. 1,2,5,6].

Moreover, another key underpinning opportunity for the AEC industry can be the added ability to explore and harness potential synergy from integrating prospective quantum computing abilities with other extant/emerging technologies (e.g. Artificial Intelligence-AI) to attain new insights, solve extant or emerging complex problems to advance the frontiers of knowledge and practice in the AEC industry.

Yet, as an embryonic area not many professionals in the AEC industry may be aware of the concept of quantum computing, its benefits and challenges. Hence, the recent developments in the area of quantum computing generate a number of questions including the following:

- What is quantum computing? And how does it differ from conventional computing?

- What is the state of relevant knowledge in the quantum computing domain?

- What can be the potential implications of quantum computing on the AEC industry?

- What are the potential challenges in harnessing the potential power of quantum computing in the AEC industry?

- What are the areas and directions for future research in this domain for the AEC industry?

This study seeks to do the following:

- Inform the AEC industry on quantum computing concepts ;

- Examine the state of knowledge and applicable technologies in the quantum computing domain highlighting key deviations from extant conventional computing;

- Investigate the potential uses and challenges of quantum computing from a generic view;

- Discuss the near term prospects and future outlook in the application of quantum computing technologies in the AEC industry.

- Explore the prospective challenges in the potential leverage of quantum computing in the AEC industry; 
- Discuss the implications of the emergence of quantum computing for research and practice in the AEC industry.

The layout of the rest of the paper is as follows. First, the paper discusses the concepts of quantum mechanics and quantum computing and addresses the unique features of these concepts that differentiate them from classical approaches. Second, the study examines certain critical milestones in the trajectory of quantum computing in areas such as Quantum supremacy and Quantum advantage. This focused examination highlights the recent Quantum supremacy claims of Google and its practical significance. Third, the study explores the competing technologies that scientists and engineers are studying to attain quantum computing goals. Fourth, the study examines the potential implications and challenges in the use of quantum computing in the AEC industry. This section also provides information on near term practical opportunities and emerging business ecosystems (at this era of technology development) that can support leverage of quantum computing in the AEC industry. Fifth, prior to the conclusions the study provides implications for practice and areas for future research on the topic in the AEC industry and allied sectors.

\section{QUANTUM MECHANICS: ROLE \& CONCEPTS}

\subsection{Quantum Mechanics: The Context for Quantum Computing}

The Oxford Learner's Dictionaries [9] defines (among others) the term, 'Quantum,' 'as a very small quantity of electromagnetic energy'. The latter meaning of this term is from the lens of the early $20^{\text {th }}$ century use of language [9]. But the term was applied in the $16^{\text {th }}$ century to refer to, 'quantity,' from its original Latin root word, 'Quantus,'(meaning how much, how great) [9]. The Cambridge Dictionary[10] provides additional perspective on the term Quantum as, 'the smallest amount or unit of something, especially energy'. Over the years the term, 'Quantum,' has been applied to nouns such as mechanics, leap, and now computers. The interest of this section is on the application of the term to mechanics and then computers.

Quantum computers (QCs) use the laws of quantum mechanics to do calculations [1]. The following sections give a brief background on quantum mechanics (on which quantum computing is based on) prior to describing what quantum computing entails.

Quantum mechanics is an area in physics that deals with the weird behavior of photons, electrons and other particles of the universe [11]. Quantum mechanics theories are underpinned by abstract rules and their mathematical depictions applied to describe the behavior of particles at very small distances and energy scales [12]. Quantum mechanics also provides theories that explain the behavior of elementary particles from both their separate and group-based perspectives [10,11,12]. And unlike the largely deterministic and intuitive nature of classical physics theories, quantum mechanics theories are based on probabilities with latent uncertainties [12].

The relevance of quantum mechanics can be established as follows. Many equations of classical mechanics stop to be useful at the very small scale level of atoms, electrons, etc.[11,12]. Quantum mechanics counterintuitively and exotically describes things at the small scale level to give accurate predictions of a wide range of observable phenomena that classical physics does not[12]. For example, whilst objects may exist in specific places at specific times in classical mechanics, in quantum mechanics objects exist in a fog of probabilities and have particular chances of being at different locations[11]. Hence, quantum mechanics results can provide strange, but relevant conclusions about the world [11] where it becomes non-feasible to apply classical mechanics concepts to explain. 
Pioneered around the turn of the $20^{\text {th }}$ century, about the same period that Albert Einstein published the theory of relativity, quantum mechanics started as controversial set of mathematical explanations of experiments which could not be explained by classical mechanics[11]. However, quantum mechanics has over the years gained acceptance via experimental verifications by a number of scientists over the decades $[11,13]$ and also significant practical recognition [14].

Quantum mechanics is now among the well-tested models that can be applied to explain the world [12,13]. Quantum mechanics confirms the same observable instinctive results expected of larger objects and it has been applied to correctly replicate classical results for larger systems[12]. Yet, its descriptions of small scale subatomic particles are exotic and non-intuitive, though accurate[12]. And very small systems are referred to as, 'Quantum systems,' when their behavior cannot be sufficiently approximated by the equations of classical physics [12]. To reinforce the significant role of quantum physics in extant daily activities the following section summarizes peculiar examples of extant practical uses of this knowledge.

\subsection{Extant Practical Uses of Quantum Mechanics}

Over the years the principles of quantum mechanics have been applied in delivering practical tools that have supported different spheres of life. For example, practical everyday applications based on quantum mechanics include the following:

- Atomic clocks, Global Positioning Systems (GPS) and Magnetic Resonance Imaging (MRI) systems [14]. GPS constellations are equipped with atomic clocks that enhance the accuracy in timing satellite signals which in turn enable smart navigation [14];

- MRI tools contain a certain key process called Nuclear Magnetic Resonance which operates by flipping the spins in the nuclei of Hydrogen atoms [14]. And this provides a picture of the practical application of quantum physics[14];

- LASERS are quantum devices applied as light source to carry messages through fiber optic cables that support telecommunications[13, 14]. LASER stands for, 'Light Amplification by Stimulated Emission of Radiation,' and this is grounded in Albert Einstein's 1917 paper[14]. Stimulated emissions occur when atoms in high energy states encounter photons of specific right wavelengths to induce them to emit identical photons to parent atoms [14];

- Modern semi-conductors depend on the band structures of solid objects and also certain critical computer tools/parts are basically quantum phenomena [13,14]. Thus, this and some of the latter/former bullets reinforce the extant role played by quantum physics in the Information and Communication Technology Industry.

Since quantum mechanics deals with how things work in nature at the fundamental level quantum computing is well positioned to leverage and explore similar systems and processes [6]. The next section examines quantum computing.

\section{WHAT IS QUANTUM COMPUTING?}

Quantum computing methods apply the unique properties of matter at the nano-scale level $[1,5,6]$. And they have the capacity to employ aspects of the near mystical phenomena of quantum mechanics to provide massive computing processing power $[1,5,6]$. Additionally, quantum computing methods require the use of outlandish computers with carefully controlled environments to do so [1]. 
Quantum bits or qubits are the basic units of quantum information [6,12]. And a quantum computer is a physical system with a collection of coupled qubits, etc. that can be controlled and manipulated to implement algorithms that give answers to specific problems (with high probability) when the system's final state is measured[12]. The qubits, etc. of a quantum computer require adequate isolation from the environment for their quantum state to be coherent for the period of a computation [12].

Comparatively, whilst conventional computers apply a stream of optical or electrical pulses in binary codes of $1 \mathrm{~s}$ and $0 \mathrm{~s}$ called bits, the basic units of a quantum computer is the qubit (or quantum bits) $[1,2,5,6,12]$. Quantum computers can be measured in terms of the number of qubits they contain, which comparatively are synonymous to the number of transistors in a conventional computer [2]. And the power of quantum computers can be underpinned by their capacity to generate and manipulate qubits $[1,2,5,6,12]$.

Qubits are characteristically subatomic particles (e.g. photons, electrons, etc.) and it is challenging to generate and manage them from a scientific and engineering perspective $[1,2,5,6,12,15]$. Hence, quantum computers were once seen as impossible feat due to their intricate need and also the need to accommodate them in unconventional environments[1,2,12].

Any two-level quantum mechanical system such as photons, electrons, ions, atomic nuclei, superconducting electronic circuits or quantum dots among others can be used as a qubit $[2,6]$. Two-level systems (or qubits) describe two energy levels and they represent the basic system in quantum mechanics and quantum technologies [16].

Qubits possess peculiar features that enable a group of connected qubits to provide more computing processing power than a similar number of conventional computing bits $[2,5,6]$. These crucial features include waveparticle duality, superposition, entanglement, coherence and measurement. The following sections describe the latter features and other appropriate characteristics of quantum objects (e.g. qubits) encapsulated in Figure 1.

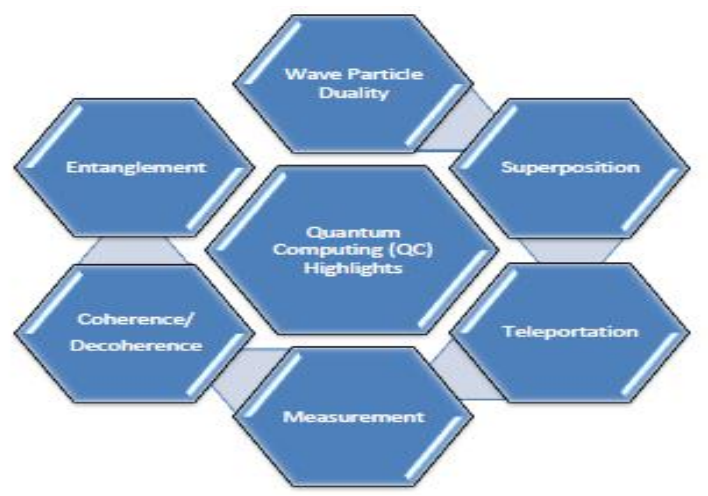

Figure 1: Quantum Computing Highlights

\section{QUANTUM FEATURES}

\subsection{Wave-Particle Duality}

Based on quantum theory, quantum objects generally do not exist in completely determined knowable states $[11,12]$. And indeed anytime a quantum object is observed it appears like a particle, but when it is not being observed it behaves like a wave [12]. Thus a quantum object possesses both wave-like and particle-like 
features[12]. This concept referred to as wave-particle duality results in many intriguing physical phenomena (e.g. superposition) [12].

Quantum objects can exist in multiple states simultaneously with each state collectively adding to and interfering like waves to define a complete or an overall quantum state generally described by wave functions $[1,5,6,11,12,15]$. But whilst the system follows a wave equation, any measurement of the system returns a value consistent with its particle-like nature [12].

\subsection{Superposition}

Superposition refers to the ability of qubits to exist in multiple states at the same time $[1,5,6,12]$. Though strange, this is a fundamental law of quantum mechanics and a key building block of quantum computers $[1,5,6,15]$. And qubits have both analogue and digital features that increase the potential of their computing power [12]. The wave function of a superposition state consists of the linear amalgamation of the contributing states, and the complex coefficients of these contributing states indicate the magnitude and phases between them [12].

Qubits can represent many potential combinations of 0 s and $1 \mathrm{~s}$ at the same time in superposition[1,2,6]. This special feature is called superposition of zero and one at the same time [1]. For example, via the use of precise laser or microwave beams qubits can be manipulated into superposition, and when many qubits are in superposition they can crunch massive numbers of potential outcomes at the same time[1,5,6]. The quantum state then collapses into $1 \mathrm{~s}$ and $0 \mathrm{~s}$ after measuring the qubits to attain the final results of the computation $[5,6]$.

It becomes a delicate state when all qubits are in superposition; as such when say any type of interference( e.g. a cosmic ray) hits the composition then the state freezes into a classical state [1,5]. Other effects that can disturb qubit operations and cause them to lose their data include temperature changes, electrical fluctuations, and vibrations [17]. Hence, one key challenge in quantum computing relates to how to maintain any calculation in the superposition state during the period of the computation cycle [1,17]. And one way to stabilize particular types of qubits is to maintain them in very cold states such as the use of special isotopes of helium to cool the qubits to about a fraction above absolute zero temperature [17].

\subsection{Entanglement}

Though scientists are now learning more about how entanglement works it is still not yet fully understood [6,18]. Qubits do not act in isolation, but become entangled to act as a group [5,6,15]. Entanglement is a unique property of some (i.e. not every) multi-particle superposition states [12]. Entanglement occurs when say a pair of qubits exist in tandem in a single quantum state and a change in the state of one of the qubits instantly leads to a change in the state of the other qubit even if they are very far apart in terms of distance with no obvious chance to interact[5,6,12]. Quantum computing leverages entangled qubits in the nature of quantum chains to deliver solutions [6].

Entanglement underpins the power of quantum computing where the addition of extra qubits in a quantum computer leads to an exponential gain in computing power [5,6]. In contrast, doubling the number of bits only

doubles the computing processing power in conventional computing [6]. Entanglement therefore facilitates the ability of quantum computers to provide exponentially dense information than classical computers[5, 6,12].

\subsection{Coherence}

Coherence is important for quantum phenomena/occurrences such as superposition, entanglement and interference [12]. In most cases the state of a quantum system can mathematically be represented as the sum of 
the possible contributing states, each scaled by a coefficient (complex number) which reflects the relative contributory weight of the state [12]. Such systems are referred to as coherent in that the participatory states can constructively and destructively interfere with each other [12]. Quantum states that are not fully coherent are represented by density matrices that define the classical probability of that system being in a specific quantum state [12].

\subsection{Decoherence}

Decoherence refers to the interactions of the qubit environment with the qubits in a manner that lead to decay in quantum behavior till they eventually disappear $[1,6,12]$. Since quantum states are very delicate, noise effects such as the slightest of changes (e.g. vibration changes) can cause them to collapse from their superposition states prior to attaining anticipated goals $[1,6,17]$.

To control quantum systems requires careful control of the system's environment via e.g. the following items [12]:

- Isolating the systems from the rest of the universe (that is very difficult to control);

- Intentional application of energy fields within the isolated region to produce a specific preferred behavior.

Yet in practice, it is impossible to completely isolate the quantum system [12]. One can only minimize the system's interactions with the environment since quantum systems over time ultimately exchange some energy with the broader environment via decoherence $[1,6,12]$. But when carefully controlled, the inherent features of quantum systems provide fresh engineering opportunities to e.g. encode, manipulate and transfer information [12].

Though quantum computers have the capacity to speed up computing power via specific orchestrated quantum algorithms, etc. decoherence makes them more prone to errors than conventional computers[5,6]. From a comparative view, classical computers are relatively reliable. For example, classical computers are very reliable at the bit level, and have fewer than one error in $10^{24}$ operations [5]. These errors in conventional computing occur more in software and mechanical malfunctioning [5]. These latter and former scenarios are in very sharp contrast to the huge engineering and scientific challenges required to avoid or even reduce errors in quantum computing systems at the moment [e.g. 1,3,5,6]. For example, in certain quantum computing applications it has been estimated that it will take about 1000 physical qubits to create a single a logical qubit (i.e. an error corrected and fault-tolerant qubit to attain reliable results) [2]. However, attaining this latter goal is not an easy feat and it is currently research in progress [2].

\subsection{Measurement and its Paradox}

\subsubsection{Measurement}

Measurement basically changes a quantum system [1,5,12]. Measurement refers to the observation of a quantum object (or quantum system-i.e. collection of quantum objects) [12]. And measurement happens when the quantum object interacts with a larger physical system which extracts information from it [12].

Measurement leads to the fundamental disruption of the quantum state [1,5,12]. And this, 'collapses,' the facet of the measured wave function into a single observable state leading to information loss [12]. The measurement represents the wave function of the observed /detected state and not that of the premeasurement state [12].

\subsubsection{Measurement Challenges}

One of the complicated features of quantum computing is that one cannot directly read the rich information of a computational state, since when extracting an answer the information collapses into discrete state with some 
probability [1,5]. And an attempt to observe a quantum system (i.e. system of quantum objects) results in observing only one of its components with a probability proportional to the square of the absolute value of its coefficient[12]. A system of $n$ quantum objects will only be observed in one of its $2^{n}$ possible states [12]. And to an observer, the system will always appear classical in nature when measured [12]. But this becomes the critical ideal amplified answer that is often sought $[1,5]$. The next section discusses other peculiar features in quantum computing.

\subsection{Teleportation}

\subsubsection{Quantum Teleportation: General}

Teleportation has over the decades generated interest from both the realms of science fiction to the domain of academic studies [19]. And decades ago, seminal academic studies such as the work of [20] anchored an influential milestone in the literature on the concept. Bennett et al.[20] showed the possibility of leveraging the support of Einstein-Podolsky-Rosen (EPR) correlations to teleport intact a quantum state from one location to another by a sender who did not know the location of the intended receiver nor the state to be teleported. And over the years scientists have applied various substrates and technologies to attain quantum teleportation in laboratories across the world[19].

Quantum teleportation entails the transfer of quantum information (e.g. particle state of a particle) [19,20,21]. Such transfers occur between distant systems without moving physical particles [19, 20,21]. Two recent research developments in teleportation can have significant influence on quantum computing applications $[18,21,22,23,24]$. These recent works have shown that higher dimensions of quantum states can be teleported $[21,23,24]$ and also that quantum teleportation can occur between two computer chips [18,22]. The following paragraphs describe the latter and former works and their implications for quantum computing.

\subsubsection{Teleportation of Higher Dimensions of Quantum States}

Recently scientists for the first time were able to teleport a 3 dimensional quantum state (called qutrit) a development which can have significant influence on the future of quantum computing $[21,23,24]$. This concept is not limited to three dimensions, but has the capacity to be extended to any number of dimensions $[21,23,24]$.

Previous work only attained the transmission of two-level state (qubits-information with values of 0 \&1) [21,24]. In classical computing, '0' and ' 1 ' represent an either/or scenario [24]. But in quantum computing these representations can exist at the same time and also anything in between is possible [24]. The recent research on the qutrit has shown the potential feasibility of the use of another third possibility of, '2' in practice[24].

The practical implications of this 3D teleportation feat include uses in future internet/communication network applications [21, 23, 24]. Thus this attainment can potentially enhance the application of higher dimensional quantum systems with the capacity to transport greater amounts of information than qubits [21, 23, 24]. In effect potentially facilitating internet connections between quantum computers with higher information capacities than what qubits previously described in the text above can provide [21, 23, 24].

\subsubsection{Quantum Teleportation between Computer Chips}

Additionally, for the first time scientists were able to show quantum teleportation between two computer chips recently by passing information between the chips using the principles of quantum mechanics rather than physical electronic connections [18]. In the two-chip teleportation experiment, the individual quantum state of a particle was transmitted across the two chips after performing quantum measurement (reaching a fidelity of 
$91 \%$ ) [18]. Each chip was fully programmed to do a range of demonstrations using the principles of entanglement [18]. This feat was attained by generating pairs of entangled photons and encoding quantum information in a manner that attained low interference levels and high levels of accuracy [18]. And Silicon chips(at micro-meter scale) constructed via the metal-oxide semiconductor process were instrumental in generating, processing, transceiving and the measurement of the multi-photon multi-qubit states in the experiment [22]. It is asserted that this work provides a potential basis for large-scale integrated communication and computational photonic quantum technologies [22]. The next section discusses certain critical milestones in quantum computing that can catalyze work in this area.

\section{CRITICAL MILESTONES: QUANTUM ADVANTAGE OR QUANTUM SUPREMACY}

\subsection{Quantum Advantage or Quantum Supremacy}

The terms quantum supremacy and quantum advantage are important concepts used in the quantum computing domain to gauge progress of quantum computers over conventional computers. Quantum supremacy or quantum superiority occurs when a quantum computer is able to do specific tasks which no conventional (transistor-based digital computer) can do within specific practical amount of time by applying practical amount of resources [2,15]. Quantum advantage: Is the superior performance of a quantum computer over conventional computing technology in terms of costs, time and quality [5]. Supremacy can be misleading and the community is now often using the term quantum advantage [2,5].

These said, attaining quantum supremacy /superiority does not necessarily imply quantum computers will be far better than all global computers in executing computing tasks [2,15]. Indeed attaining quantum supremacy /superiority is a distinguished achievement, but this feat will not necessarily translate into instant or near-term transformational changes in the computing world [2].

Though quantum superiority is conceptually important, it can probably be applied to solve a computing problem with little or no practical significance [2]. Hence, the belief of many is on attaining a quantum advantage - a more beneficial feat when quantum computers can do useful work that no conventional computers can solve in practical time frame in a practical cost effective manner [2,3,5].

To place this in context, in a recent IBM internal analysis based on polynomial and exponential functions the following comparative times were deduced in terms of the time to solve problems via a classical algorithm with exponential runtime and quantum algorithms with polynomial runtimes[3]:

- A problem that will take a classical computer 10secs, 2 minutes, 330 years, 3300 years, and the age of the universe to solve could be done respectively within 1 minute, 2 minutes, 10 mins, 11 minutes and 24 minutes using a quantum computer.

Thus, based on this data quantum advantage can for example be demonstrated from the locus when the quantum computer can do work that will take about 330 years by a conventional computer to complete.

\subsection{The First Claim: Google and Quantum Supremacy}

Recently scientists from the Google team claimed attainment of quantum supremacy. This is a much anticipated milestone in quantum computing [15,25]. Google's team claimed that their quantum computer performed a specific computation that is beyond the abilities of regular conventional computers [15]. The claim asserted that it would have taken even the best classical supercomputer an estimated time of 10,000 years to complete the same computation [15,25]. Thus even on a classical computer with 1 million procession units (about 100,000 desktop computers)[15]. But Google's quantum computer (Sycamore) took only 3 minutes 20 seconds to do this task [15]. Google's solution applied 53 qubits since 1 of Sycamore's 54 qubits was broken [15]. This generated $2^{53}$ possible combinations of strings of $1 \mathrm{~s}$ and 0 s [15]. 
Though the calculation performed by the quantum computer (i.e. checking outputs from a quantum random generator) may in itself not be practically significant, the scientific importance of this feat is significant [15]. However, IBM has disputed the Google team's claim of attaining quantum supremacy by asserting that the said problem solved by Google's quantum computer could be solved in 2.5 days applying a different classical technique [15]. And hence not the 10,000 years as claimed by Google's team [15].

However, other experts argue that even if IBM's position is correct, the Google team's work is still significant since it has proven quantum advantage by showing how a quantum computer can perform faster than a classical computer [15]. Additionally, since Google's algorithmic application run on a 53 qubit computing this was only a fraction of the one million qubits that will be needed to operate a versatile general purpose machine [15]. And the hope is that quantum computers may at some time in the future run revolutionary algorithms to e.g. factor large numbers for encryption or search unwieldy/unmanageable databases [15] though such general purpose machines are deemed to be decades away [12,15]. The next section discusses types of quantum computing approaches.

\section{QUANTUM COMPUTING TECHNOLOGY TYPES}

\subsection{Types of Technologies}

A number of technologies are being used or examined to produce useful quantum computers. They include the use/examination of Superconducting qubits, Ion Traps, Silicon-based qubit technology, Topological quantum approach, Photonic quantum computers and Quantum annealers. Of these the perceived leading technologies are the Superconducting qubits \& Ion Traps[e.g. 5,12]. The following sections discuss the technologies:

- Superconducting Qubits: Is a two level energy system of superconducting circuits that forms a socalled transom (a somewhat noise resistant qubit) [2,5,12]. Large technology companies are leaning on superconducting circuit quantum computers because they are based on standardized Complimentary Metal-Oxide Semi-Conductor (CMOS) Technology and they are relatively easier to handle [5]. Examples of entities using or examining the concept include Google, Intel, Rigetti, Alibaba \& Quantum Circuits [5]. Measurement times for known error-correction methods are in the range of microseconds [5]. The research in this area is embryonic and faces many scientific and engineering challenges [5].

- Ion Traps: Typical example of the basic elements of this system relates to single ions (charged atoms) trapped in say electric or magnetic fields $[2,5,12,17]$. The qubits are formed from the energy levels of their inherent spins [5]. Ion trap technological approach requires deep knowledge of lasers and optics [17]. And Ion traps face huge challenge when they reach 50 qubits, where connecting another trap becomes difficult [5].

Others quantum computing technologies include the following.

- Silicon-based Qubit Technology: This technology is work in progress and can in the long term make the scaling of atomic size qubits easier and faster; this approach can also draw on the extant global silicon manufacturing experience [5,17]. The concept of applying silicon-based CMOS technologies to develop quantum computers was initially proposed by Bruce Kane in 1998 [26]. Silicon provides a relatively noise-free environment for spins to retain their quantum nature [26]. Purification methods can be applied to remove unwanted isotopes (a source of quantum bit errors) prior to producing silicon crystals to give robust solid state qubits[26]. Following Kane's seminal work, scientists have developed silicon-based quantum technology, and shown that single-qubit and two-qubit logic operations can be done by applying phosphorous atoms [26, 27]. Also recent work at Delft University of Technology has shown the possibility of controlling entangled silicon-based qubits with adequate 
precision [26]. Key challenges of the silicon-based approach include how to overcome the variability in the features of quantum devices [26].

- Topological Quantum Approach: Three scientists won the 2016 Nobel Prize in Physics for their early theoretical work on topological states of matter [28,29], but work on the topological quantum approach is still in early stages $[5,13]$. Topological quantum computing approach seeks to make quantum systems immune to the usual/typical sources of quantum decoherence and as such make decoherence irrelevant in quantum systems rather than noiseless[13]. The topological quantum approach is a low error rate approach (e.g. 1 part per million or even billion) [5,13].

From an industry perspective, Microsoft is one of the major players in the research or use of the topological quantum approach [29]. Whilst IBM, Google and other academic labs (for instance) have selected a relatively mature technology in superconducting wires to make qubits it is perceived that Microsoft has taken a more difficult path than its rivals[29]. The company has been working on this concept for over a decade and is currently developing devices and software for future machines [29].

Through the topological quantum approach, Microsoft seeks to encode qubits in a sort of quasiparticles (called non-abelian anyons) which are particle-like objects which materialize from interactions within matter [29]. Anyons act as electrons and their anti-matter counterparts at the same time and are fuzzy than normal particles [29]. They can be measured with high precision devices, but they are not perceptible under any microscope [29]. Information is not encoded in the quasiparticles themselves, but rather through a concept called braiding (the order in which the position of the anyons are swapped) [29]. Thus the information is encoded in the 'topological' collective property of the system [29]. A key advantage of the topological computing approach is in the potential to avoid doing extensive and expensive qubit error correction[29].

Though some scientists think that the key underpinning particles in the topological approach might not even exist [29], Microsoft however expects to exploit topological properties (that render quantum states very robust to external interference) to develop topological quantum computers [29] in the future.

- Photonic Quantum Computing: Scholars have recently been able to develop a photonic quantum processor that can produce and deploy two qubits encoded in photons to do for example two-qubit calculation [30]. Foremost competing tools to the photonic quantum approach such as trapped ions (e.g. held in place by lasers) and superconducting wires (frozen to near absolute zero) face decoherence problems that can ultimately render them ineffectual as the number of qubits in the system increases [5,30]. But since photons do not interact with the environment decoherence is not a key issue in the photonic approach [30]. Additionally photons can be deployed in an ultrahigh precision manner and also the use of photonic chips can draw on the known extant silicon industry experience [30]. Furthermore, the photonic approach can have the advantage of operating at room temperature [5].The major challenge for the photonic technology is in developing single photon sources and detectors [5,30]. Additionally, the approach faces the challenge of controlling multiphoton interactions that are crucial for two-qubit gates[5]. 
6.2 Quantum Annealers (the Odd Technology)

D-Wave Systems pioneered the use of the first quantum computer on the market [1]. The company applies a Quantum annealer which is a special purpose machine focused on solving optimization problems by finding lowest point in a high-dimensional energy 'scape'[5]. The Quantum annealer is not a circuit-based quantum computer and successfully solves optimization problems using different methods than the use of quantum circuits [31].

Some scholars have argued that there is no extant persuasive evidence to show that Quantum annealers can actually solve problems (i.e. the same problems) quicker than the best classical computing tools [31]. Additionally, certain scholars have questioned how D-Wave Systems' approach fits into the quantum computing domain [e.g. 5]. Yet, some scholars believe that in the near term the company is still a key player in the market [5]. And research is now also focusing on Non-Stoquastic Quantum Annealers that have the better potential capacity to do better than what classical algorithms can do [31].

Quantum Annealer Operational Environment: The environment in which the quantum computer systems operate is at a temperature near absolute zero (-273 degree Celsius), thus the lowest temperature that is physically possible in the universe called the thermodynamic limit [1]. The quantum machines run at $0.01 \mathrm{~K}$ degrees or 10 degrees millikelvins [1].Additionally, the systems require the creation of magnetic vacuum and air vacuum [1].

\subsection{Noisy Intermediate-Scale Quantum (NISQ) Tools}

One ultimate or long term goal in quantum computing research is to attain Fault-tolerant universal quantum computers [e.g.3,31]. In the near-term, Noisy Intermediate-Scale Quantum(NISQ) computing gives a potential option for quantum computing use, as advances ultimately move towards Fault-tolerant universal quantum computers that can be exponentially faster than classical computers [3].

NISQ is 'Noisy' because of the lack of control on the qubits and the effect of noise in the system that can cause significant limitations on what quantum computers can attain in the near term [31]. The 'Intermediate-Scale' in the acronym relates to the size of quantum computers that will be available in the near term with qubits ranging from 50 to a few hundreds of qubits[31]. It has been suggested that the number 50 is a significant number for the reason that this is the number that provides an ability beyond what can be simulated by the most extant powerful digital supercomputer [31]. NISQ is an intermediate step towards attaining transformative quantum computers that are decades away [3,12,31]. NISQ tools are not yet fully ready to contest with the best classical computers, but certain experimental results provide the probability that this can occur in the future [31].

To sum Figure 2 captures the major quantum computing technologies that have received attention in research and practice in recent times and the context of the near-term and ultimate goal of such efforts to attain faulttolerant universal quantum computers. Also included in Figure 2 is the notion of the possibility of emerging radical or evolutionary technologies that can emerge over time to accelerate the attainment of the latter ultimate goal. 


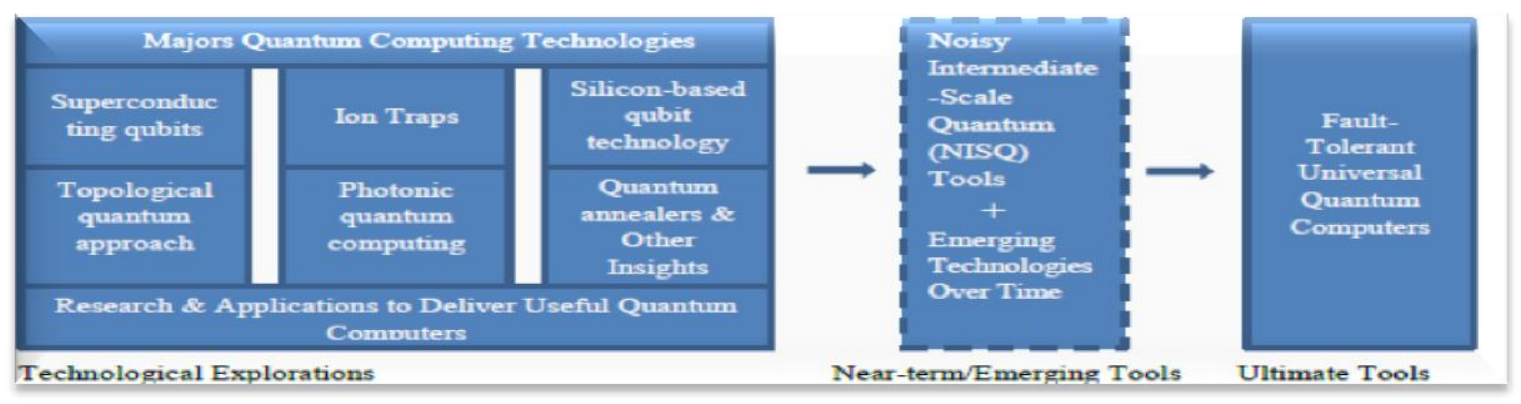

Figure 2: Context of technological explorations, near term/emerging tools/ultimate quantum computing tools.

The next section provides a brief overview of parameters that can be helpful in understanding the potential utility of quantum computers. It must be noted, however, that quantum computing is still an emerging area and hence these tools are solely for cursory comparative overview of quantum computing tools.

\subsection{Technical Assessment}

From a technical perspective it may be important to assess the utility of potential quantum computing tools. The following are certain distilled items that can be applied to support quick performance (and other) evaluations. These criteria are however, skewed for circuit based quantum computing since though there are several quantum computing technologies most of them are at relatively embryonic phase for any meaningful suggestions. The following examples stated for the current state of quantum computing technologies are primarily for circuit based quantum computers. The parameters are as follows [2,5]:

- Size: Current technologies base the size of quantum technologies on the number of physical qubits. Physical qubits are important for scaling considerations [5]. Size issues will in future be likely determined by the number of fully error-corrected qubits called logical qubits [5].

The capabilities of quantum computers to do calculations can be assessed on the basis of variables such as the following [5,17]:

- Qubit lifetime: Period of time information can be stored in a qubit. This shows how long qubits can store and process information. The known lifetime of a qubit is currently between 50 microseconds to 50 seconds for current gate-based quantum computers.

- Gate fidelity /Operational accuracy: applies to the accuracy of a 2 qubit operation. And this is a key indicator of the quality and overhead for quantum error correction. Current range is between 90-99.9\% for current gate-based quantum computers.

- Gate operation time: is the time utilized for a two qubit operation. It indicates the speed for manipulating physical qubits. This is between $1 \mathbf{n s}-50$ micro-secs for current gate-based quantum computers ;

- Connectivity: refers to the connection between qubits and they indicate the extent of information that can be encoded in a qubit group state. Due to the importance of entanglement in quantum computers, this requires qubits to be interconnected for interaction. Connectivity ranges from one-to-one(1:1) connections to the preferred all-to-all $(n: n)$ connections.

Though narrow at this stage, it is hoped that leveraging such parameters can help provide cursory comparison of appropriate extant quantum computing tools. The next section examines the potential impact of developments in the quantum computing area on the AEC industry. 


\section{THE ARCHITECTURE, ENGINEERING AND CONSTRUCTION (AEC) INDUSTRY AND THE QUANTUM COMPUTING WAVE}

\subsection{Context}

The AEC industry accounts for a significant aspect of the economies of many countries [4]. The industry plays the crucial role in many places by providing the infrastructure that is applied by the other sectors of national economies to generate more value (in terms of products and services) [4]. Hence, targeted investments in constructed products and services yield the crucial catalyst that can drive growth in many economies.

However, construction products are in most cases durable goods [e.g. 4] and they require huge capital investments and longer times to produce. Efficiency and effectiveness in product delivery in the AEC industry is therefore a critical issue in many resource constrained locations. Among others, radical innovation or continuous product and process improvements in the industry are some of the required essential areas that have been identified over the years to attain the latter goals of efficient and effective AEC industry product delivery to fit the needs of value provision within the parameters of any contextual investment constraints $[4,8]$.

Yet, over the years the AEC industry has been consistently criticized for lacking the drive and agility to adopt or adapt new technologies to improve value delivery [e.g. 4]. In most cases and in many places the AEC industry has collectively lagged behind other sectors (e.g. manufacturing and aeronautical industries) of many economies in adopting fresh technologies to improve productivity and also significantly transform overall value delivery [e.g. 4].

The latter sketch is not intended to preclude mention of recent efforts and drives in specific AEC industry markets to innovate and improve products and services in the industry. A typical example of this is in the recent efforts in many places e.g. to apply Virtual construction, Building Information Modelling (BIM) [32, 33,34] and other recent technological advances in Artificial Intelligence (AI) and other digital tools to reinvent processes and product delivery to meet specific value delivery goals.

Yet, significant cultural shift (e.g. in technology leverage) across the industry will be necessary over time to transform AEC industry value delivery mechanisms to be in sync with innovative developments in many other sectors of many economies that rely on the products and services of the industry. Major leaps in certain key technologies accepted and used across other industries often drive (in a significant way) the imperative need for AEC industry changes. A typical example is in the structural shift from manual processes to the wide adoption of digital computers and allied digital information and communication tools to enhance efficiency in value delivery. Though the AEC industry was relatively slow in accepting and adopting these tools [e.g. 4]they have now become significant imperative tools in the industry.

\subsubsection{AEC Industry \& the Growing Interest in Quantum Computing}

Interest in quantum computing is currently on the rise since the concept is perceived to possess immense potential over digital computing in specific aspects $[2,3,12,15]$. And the potential influence of quantum computing is likely to cut across all industries [e.g.2,3,5,12] including the AEC industry. The AEC industry will also likely play a central role in building basic infrastructure required to implement the quantum computing technologies across industries.

Additionally, specific potential breakthroughs in the quantum computing sector can unleash significant security risks for conventional security systems and extant encrypted confidential data [2,5]. It is therefore 
important for entities in the AEC industry to monitor developments in the quantum computing research domain to proactively prevent harm that can arise from inaction.

From a time spectrum perspective, tracking abilities in the quantum computing domain over its technology maturity lifecycle can provide immediate, medium-term and long-term opportunities for AEC industry related companies as the research and practice (e.g. in the science, engineering, and quantum computing technology management) evolve over time. For example, in the near term quantum computing technologies such as Noisy Intermediate -Scale Quantum (NISQ) systems can be available for specific business case uses and exploration. The next sections in this chapter discuss the potential prospects and impacts of developments in the quantum computing domain on the AEC industry. First, the near term prospects for actors in the construction industry to explore the practical use of quantum computing tools are examined. Then this is followed by a generic outlook of the impact of quantum computing in supporting business management, project management and asset management.

\subsection{Practical NISQ Use Prospects And Extant Quantum Computing Ecosystem}

\subsubsection{Near Term Practical NISQ Use and Other Prospects}

A number of small quantum devices now exist and most of them are small, noisy and not significantly powerful as extant classical computers [35]. Yet they are gradually advancing into the next generation of devices [35]. And cloud-based quantum computing has been applied, for instance, to test machine learning algorithms [35 ]. Allied to this situation is the availability of a number of quantum computing software and simulators based on a variety of classical computing languages [35]. Quantiki [36] for instance provides a detailed list of some of the quantum computing simulators grouped under a number of programming languages such as C++, Java, Javascript, Mathematica, Maple, etc. The key development here is that such initiatives have now opened up opportunities for many potential users of quantum computing to explore and experience the use of quantum computers. And construction industry-based professionals can also do so now.

The recent emergence of a number of quantum computing technologies has also prompted the need for certification and benchmark characterization of quantum devices to evaluate/ guarantee their correct functioning [37]. And work in this area is showing significant promise. For example, Cycle benchmarking is an approach tailored to enhance the fair evaluation of the value which competing quantum processors provide [37]. The approach is a scalable protocol for characterizing local and global errors across multi-qubit quantum processors [37]. Via this method quantum computers can be compared and also applied to determine which quantum processor architectures are very error prone (for development) or are worthy for scaling up [37]. Additionally this approach can help to develop performance standards to support efforts for building large scale practical computers[37]. And the approach can be applied to quantum computing solutions across platforms for users to evaluate the probabilities of errors in the solution [37]. This development can in the near term give AEC professionals the ability to make better informed decisions in the application of these quantum computing tools.

Moreover, one critical jewel of knowledge will be the ability to understand the extant and evolving quantum computing ecosystem to enhance the ability to tap into potential opportunities and also build effective capacity over time. The next section examines trends in this latter ecosystem.

\subsubsection{Quantum Computing Ecosystem}

In the near term, understanding the structure and dynamics of the following emerging quantum computing ecosystems will be a useful resource to leverage for capacity building. The example structure provided below 
covers areas such as hardware and systems players to end-to-end quantum computing providers with examples of companies actively engaged in the indicated areas [2,3,5,38, 39,40]:

- Hardware and systems actors: e.g. IonQ, Intel, Quantum Circuits; QuTech; BraneCell;

- Software and services actors: 1Qbit, QxBranch; QCWare; Zapata Computing; Cambridge Quantum Computing;

- Specialists: Silicon Quantum Computing; SeeQC; Entanglement partners; QbitLogic; Qunidom; QCTRL; Quantum Benchmark; Strangeworks;

- End-to-end providers: D-Wave Systems; IBM; Google; Microsoft; Rigetti Computing; Alibaba Group; Honeywell.

It is important to note that the above ecosystem information reflects specific time and can evolve and change over time as quantum computing technologies mature. Consolidations, demise and emerging new players are not impossible in such scenarios over time. Yet, some of these data can be a good starting place for exploring opportunities, building partnerships or choosing potential technology trajectory. For example, there are a number of open-based efforts including notable cloud-based quantum computing initiatives by entities such as IBM Qiskit, Forest, Project Q, Quantum Developer Kit(Q\#) [3,35].These opportunities can be a good starting place to explore the power of quantum computing for simulation, educational and other purposes in the AEC Industry.

After building capacity on specific quantum computing awareness, knowledge of the explicit maturing capacities of quantum computing tools over time as well as the potential use cases in specific AEC industry related businesses or project delivery can be essential. These steps could be framed within the context of bigger strategic and tactical perspectives that cover specific areas such as the following: Quantum computing (QC) vision \& mission, QC planning, compiling QC business and technical use cases, QC implementation, capacity building; sustaining QC use; QC technology monitoring and continuous improvement; building strategic partnerships to leverage QCs, etc.

At the tactical level deepening relevant knowledge of quantum computing -related capabilities over time and applying specific use cases from the bigger frame of competitive space can be productive. Over time relevant tactical uses can be potentially distilled effectively from quantum computing tools as their practical uses in AEC industry business management and AEC industry project delivery become more defined/matured.

\subsection{Generic Outlook and Opportunities}

\subsubsection{Business and Project Delivery Prospective Opportunities}

The potential opportunities from quantum computing can be examined from the perspective of business management and construction project delivery. For most AEC industry-based businesses the two areas (i.e. business management and project delivery) are intertwined since construction oriented businesses often deliver tasks via projects. But the two areas are distinctly identified in this section to enhance the discussion.

AEC industry-based businesses often cover a range of strategic areas to remain relevant and also grow. From a business management view, these opportunities relate to the potential prospective benefits that can accrue over time through quantum computing to the core business operations of entities in the AEC industry.

Examples of potential tactical uses from a business view that may evolve over time include areas such as the following: Building secure environments for business applications; applying quantum-based simulations in tendering or procuring work; leveraging quantum-based simulations in business process engineering to enhance profitability; building human resource capacity; optimizing tax planning and taxation solutions , 
accounting and financial planning via use of quantum-based tools ; business scenario planning, marketing solutions, etc.

From a construction project delivery perspective, the opportunities concern the prospective benefits that can be potentially gained in construction project delivery from quantum computing-based systems. Key potential benefits from advances in quantum computing are likely to be significant in the delivery of complex construction mega-projects, construction programs and works in project finance (e.g. in Public-Private Partnerships). These types of works entail significant risks, uncertainties and many complex variables that often affect project success. Over time potential advances in quantum computing power and relevant quantumbased algorithms can be applied to explore challenges often faced in these types of complex works to augment decisions to enhance delivery success.

Projects and programs can also benefit from potential better data encryption, enhanced security and communication that quantum computers have the potential capacity to provide [e.g. 3,12]. This can potentially curb data breaches, preserve confidentiality and privacy of actors in projects and programs. Construction project delivery also entails generation and use of massive data in certain cases. Increased capacity through quantum computing can enhance such data management and manipulation.

Quantum computing is still incipient work in progress with huge potential influence[e.g. 12]. But the most likely near-term impact and potential opportunity from developments in quantum computing will be in the area of computer security systems [e.g. 2,5,12]. Quantum computing systems have the capacity to disrupt extant digital cryptography [2,5]. But this threat also provides the opportunity for AEC industry related companies to strengthen their communication security systems via quantum-based techniques to guard against potential hackers who may be seeking to exploit any potential security flaws [e.g. 2,5,12].

Extant digital computing tools have limited capacity in solving certain complex problems via simulations on classical computers [12,31]. From a long-term view, quantum simulations on quantum computers can potentially have significant influence in many areas of life [12,31]. Hence, another area of opportunity for AEC industry-based businesses will be the potential to apply quantum related simulations and sampling to explore complex business problems that are currently difficult to solve. For example, procurement is at the core of winning projects and sustaining business survival in the AEC industry. Improved computational power can support businesses to explore and find better ways to compete and win tenders; solve difficult customer related business problems; prepare better proposals and explore construction financing issues to create better opportunities for business.

The potential unique abilities of quantum computers can be explored and integrated into other problem solving tools to increase and deliver fresh synergistic business solutions. Examples in AEC industry related business solutions include harnessing potential increased computing power from quantum computing to power aspects of AI (e.g. Machine Learning) to augment business decisions and operations in say attaining better winning bids without compromising too much profit margins in very tight bid competition. Also extant technologies in areas such as Big data, Internet of Things (IOT), Digital Twins, BIM, and Blockchain technology can over time be supported with the unique potential abilities of quantum computing in an integrated manner to provide better insights into areas that may otherwise be limited by current computing capacity. In effect from a business perspective the generic opportunities that may be available to most businesses across industries will also be available to AEC industry related businesses to explore. 
7.4 Asset and Property Management

Constructed products enter the operations and maintenance phase after delivery. This is a phase that users experience the real benefits of the delivered product. Synergies generated across the unique potential abilities of quantum computing and tools that enhance property management (e.g. Digital Twins, IOT, etc.) can produce productive benefits. Additionally, prospective increased security via quantum computing [e.g. 3,12$]$ can enhance data security, avoid the hacking of connected IOT, and boost privacy. Property managers can also use potential quantum-based simulated abilities to deal with logistics, maintenance planning and execution issues to maintain environmentally sustainable goals. The next section discusses the potential challenges concerning quantum computers and their potential use in the AEC industry.

\subsection{Challenges}

There are a number of challenges relating to quantum computers and their potential use in the AEC industry. The following items chronicle of some of them.

- Quantum computing technology is still in the early stages of research and development. There are still outstanding key scientific and engineering issues with the technology including e.g. dealing with decoherence, high error rates in producing qubits via certain methods and the significant challenges in correcting these errors [12,31].

- Quantum supremacy has potential implications for quantum computing research, cryptography and cryptocurrencies whose security depend on random keys [15,25]. Google's team has already claimed a disputed Quantum supremacy [15,25]. Yet, attaining supremacy does not mean instant access to general purpose universal quantum computers [15,25]. Future work is still required to demonstrate that programmable quantum computers can solve useful tasks that cannot be feasibly solved in any other way $[15,25]$.

- Quantum supremacy in error correction is also needed - thus a method to correct noise-induced errors that have the tendency to wreck calculations [15,25]. Experts believe that such an attainment is important to scale the functioning of quantum computers[15,25].

- Scholars caution that there is a long way (decades) before any general purpose quantum computer can be produced and become universally available for general application [12,17, 31]. One will require general or universal quantum computers to solve far larger and wider problems [2,31]. And the latter scenario will need hundreds of logical qubits created from hundreds of thousands of physical qubits to do so [2]. This is still an embryonic research in progress [2, 15, 25].

- Certain experts speculate that quantum computers are likely to be large, non-portable tools that will likely cost millions of dollars and also require experts to run or program [2]. Additionally, they conjecture that they are more likely to be better than conventional computers in certain limited areas required to solve hard computing problems [2]. Yet, with the many ongoing research fronts on this concept it is not that simple to settle on such an idea. For example, digital computers also started as very huge machines, but evolved over time into desktops, laptops and the present day pocket-size smart computers. And to place this in context, the computational power of today's pocket-size smart phones is similar to that of the fastest supercomputers 20 years ago [12].

In spite of the above challenges the following developments are also worthy of note: Hybrid simulations

- Apart from the potential intrinsic relevance of quantum computers, advances in this area have enabled the use of classical computers to simulate quantum techniques [2]. And these strides are important prior to the attainment of the full potential of general purpose quantum computers [2]. 
Quantum safe security

- Quantum computers can potentially be applied to break through extant security codes that are undecipherable [2]. Potential advances in quantum computing communication systems therefore require business entities (including AEC industry related businesses) to prepare their communication systems to deal with this potential threat. That is business entities should prepare to make their extant digital systems quantum proof to be quantum safe against potential threats in the near term [2].

Though the quantum computing domain is still embryonic, a key challenge for the AEC industry is in extant attitude to embracing new technologies. Building awareness and capacity to match the evolution of quantum computing tools is an essential step in running with the appropriate trends. This approach can over time avoid creating huge knowledge gaps on relevant matters. Also although the state of technology maturity is in the long term anything can happen in research and the unexpected can be hastened for quantum computing breakthroughs.

\section{IMPLICATIONS FOR AEC INDUSTRY PRACTICE AND RESEARCH}

\subsection{AEC Industry Practice Outlook}

Over time, the need for quantum computing will vary across the AEC industry. Based on previous technology adoption trends the following can be deduced. Not all companies have adopted the depth of digital computing and allied ICT potential even with extant computing abilities. The need for new technologies such as quantum computing over time will depend on awareness; extant capacity gaps; cultural inclinations ; trends in the behavior of competitors in the industry; driven influence of major clients; the draw of the quantum computing technology, and so forth.

Prospective tools in quantum computing over time can radically disrupt extant tools or augment current technologies used by firms in the AEC industry. At least in the near-term hybrid infusion of quantum techniques in areas where they can perform better than conventional tools (e.g. in optimization studies/simulations) can augment tactical areas where companies are already doing well in or are lacking. For example, advanced adopters of Building Information Modelling (BIM) can benefit from near-term hybrid infusion of quantum techniques. And also the weak users of extant technologies who have the drive to improve systems and processes can leverage potential hybrid quantum-based techniques to strengthen activities.

At the appropriate time, extant digital technology laggards can also have the opportunity to surge ahead of the opportunity curve by coasting on the crest of potential quantum computing-based technology disruption(if it occurs) to improve potential computing power and also allied potential complex problem-solving abilities in any emerging new paradigm( that is likely to be better than the preceding era).

To technology skeptics it can be another vindication when the promise of quantum computing fails to match touted expectations. Yet, the converse is also true. For example, when (as touted) significant quantum computing-based advances occur to build on extant momentum to completely disrupt digital computing and allied communication technologies. At least one key area that buttresses the latter sway is likely to be in the area of computer network security which can potentially be cracked via the use of demonstrated quantum techniques to severely compromise extant confidential information assets that can lead to significant business losses [3,12,31]. Additionally, quantum computers are potentially better placed than conventional computers to perform quantum-based simulations required for specific studies[17,12,31].

\subsection{Educational Institutions}

Educational institutions can design courses tailored to specifically fit quantum computing uses in the AEC industry to build potential capacity. This drive can incorporate capacity development for learners to provide 
specific algorithms to fit/solve complex AEC industry problems. These initiatives can boost awareness and capacity in the industry to facilitate quantum computing adoption at the appropriate time on the technology maturity curve. Partnerships with companies in the quantum computing ecosystem can offer practical 'co-op' experience to prepare students for the future. For example, as already indicated open source efforts/quantum initiatives such as IBM Qiskit, Forest, Project Q, Quantum Developer Kit(Q\#) [e.g. 3,35] among others can provide typical opportunities.

Over time combined practical and theoretical research approaches focused on specific potential AEC industry benefits appropriately linked to the broader research domain in quantum computing can build needed capacity. AEC industry research teams can also benefit from cross-disciplinary research especially in institutions with established or advanced research skills in quantum computing.

\subsection{Professional Associations}

Various professional associations in the AEC industry with discipline specific focus can also help build capacity via continuous professional development initiatives and credits. They can partner with credible institutions to fittingly explore and advance their professions. And also help build capacity by sponsoring discipline specific research in relation to quantum computing; support building practical discipline-specific quantum computing uses, frameworks, methodologies, tools and databases. These initiatives can start with building awareness and continuously doing so over time to match the quantum computing technology maturity cycle.

\subsection{Policy Makers}

Policy makers should stress the synergies that can be attained via incorporating broader industries (including AEC industry) in quantum computing research and diffusion. And if necessary support appropriate activities such as funding, building use cases /case studies $\&$ best practices, models, etc.

Other critical areas include building standards to guide practice and industry applications. Extant Standards authorities /bodies will require extensions to extant work to incorporate quantum computing techniques over time as technology matures.

\subsection{Areas for Future Research Focus}

There can be a number of research opportunities based on extant and emerging revelations or developments in the quantum computing domain over time. These opportunities include the following.

\subsubsection{Architectural Designs}

To push the boundaries of design bold architectural initiatives such as regenerative design concepts that can have very positive impacts on the environment are essential. Material science can play a significant role in this area as designers seek fresh materials aside the traditional ones to provide positive rather than negative or zero impact on the environment. Cross-disciplinary research including multi-disciplines such as architects, engineers, biologists, material scientists, chemists, physicists, etc. can apply the emerging potential computing power of quantum computers to examine ways to develop fresh materials to support regenerative designs. For example, such teams can simulate/reengineer molecules [e.g.3,17] and other material properties to extend the spectrum of environmentally positive material choices that architects can select from to produce better regenerative buildings with positive impacts on the environment. Tracking the maturity cycle of quantum computers over time will be essential in generating appropriate algorithms that can support such drives. 


\subsubsection{Engineering Designs}

There are different aspects of engineering works required in construction works (e.g. structural, electrical, mechanical, acoustics, highways, geodetic, traffic, materials, etc.). Just as discussed under architectural designs these disciplines can also benefit from potential advances in quantum computing to advance the frontiers of design. At the appropriate time, prospective increased computing power and potential quantumbased algorithms can be leveraged to explore and extend frontiers of knowledge in applicable engineering theory and practice. Especially, in areas where extant conventional tools lack the power to do so. Possibilities can be in areas such as developing fresh materials, new engineering methods and techniques that improve on extant ways of construction or dynamically change extant paradigms e.g. to support regenerative designs. Yet again research integrating quantum computing and other extant tools (e.g. AI, BIM, IOT, etc.) can be suitably leveraged to extend the boundaries of knowledge.

\subsubsection{Software and Algorithms}

Over time research and development of AEC industry-focused software and algorithms to operate on quantum computers and quantum computing networks will be a significant need as quantum computing-based tools become more practically defined. The open source software, etc. approach can help speed up specific targets in this domain. After building enough capacity in this domain, tailored tools can then emerge to deal with basic and very complex AEC industry problems via quantum computing-based tools over time. Other alternatives include forming research partnerships with entities from quantum computing ecosystems to explore AEC industry specific problems. Opportunities exist especially for AEC industry scholars in academic institutions which are well grounded in quantum computing research. Forming cross-functional/cross-disciplinary research teams to study AEC industry related problems can go far in attaining such goals. And Figure 3 distils a pictorial model extracted from the text that can be applied to focus areas for deeper exploration as discussed in the text.

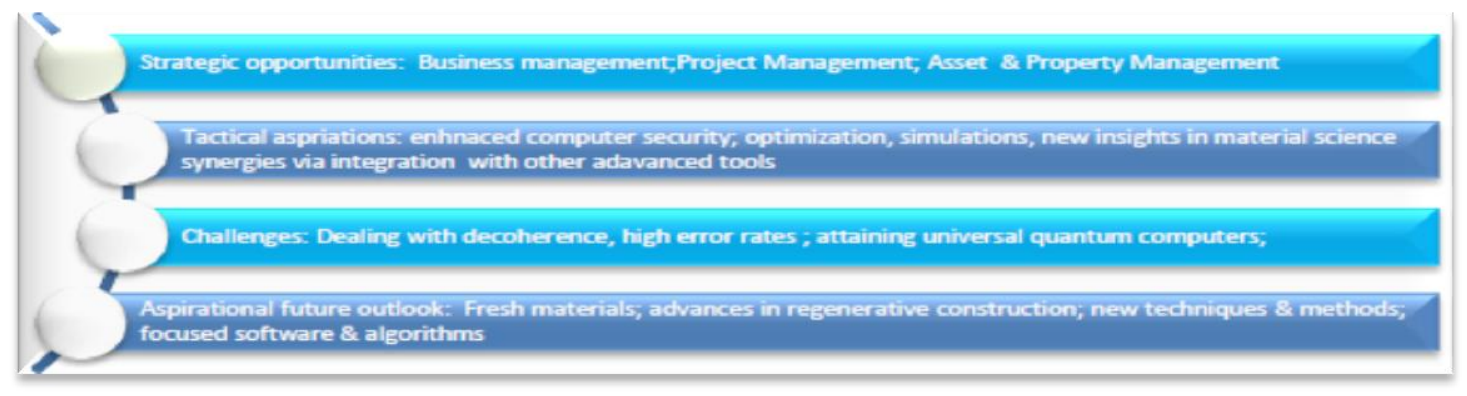

Figure 3: Distilled Highlights

\section{CONCLUSION}

Businesses across industries including those in the AEC industry face extant and emerging complex problems that are difficult to solve via the use of extant computing power. Additionally, the capacity of extant digital computing power are fast reaching their ultimate capacity limits.

Quantum computing is an incipient and evolving domain that can provide another path to exponentially increase computing power to deal with some of the complex problems that conventional computing tools are limited in solving. Potential advances in quantum computing can increasingly influence many aspects of life including the AEC industry. But not many studies have been done in the AEC industry related literature to build awareness and explore the potential impact of quantum computing on the AEC industry. 
This study has illuminated the concept of quantum computing; the state of knowledge of the concept and associated challenges; the potential impact of the concept on the AEC industry over time; and also proposed implications for research and practice. Quantum computing possesses potential capacity to increase computing power, enhance security of computing networks, and provide the platform to solve complex problems(e.g. optimization issues, simulate options in mega-projects, etc.) in the AEC industry. And in the near term a number of prospects exist for actors in the AEC industry to alternatively explore practical solutions to extant difficult problems via open-sourced quantum- based initiatives.

Yet, quantum computing technology faces a number of scientific, engineering, and practical implementation challenges that may take years to make the technology universally available for use across industries.

Examination of the topic is however important for the AEC industry to keep in the loop and build appropriate capacity over time to deal with knowledge gaps as the technology progresses over time. This approach can be a strategic logical way for actors in the AEC industry to position themselves to effectively leverage the potential benefits of quantum computing and also deal with its prospective challenges and risks.

\section{REFERENCES}

[1]Brownnell, V., 2016. The growing potential of quantum computing. McKinsey \& Company.

[2] Stewart, D.,2019. Quantum Computers: the next supercomputers but not the next laptop. Technology, Media \& Telecommunications Predictions 2019. Deloitte Insights.

[3] Gil, D., Mantas, J.,Sutor,R.,Kesterson-Townes,L, Flother,F., and Schnabel,C.,2018. Coming soon to your business-Quantum computing-5strategies to prepare for the paradigm-shifting technology. IBM Institute for Business Value, IBM. Retrieved September 24, 2019 from https://www.ibm.com/thought-leadership/institutebusiness-value/report/quantumstrategy

[4] Barima, O., 2017. Leveraging the blockchain technology to improve construction value delivery: The opportunities, benefits and challenges. In Hall, K. (Ed.), Construction projects: Improvement strategies, quality management and potential challenges, (Chapter 3,pp.93-112), New York: Nova Science Publishers, Inc.

[5] Gerbert, P. and Ruess,F.,2018. The next decade in Quantum Computing-And how to play. Boston Consulting Group.

[6] Giles,M.,2019. Explainer: What is Quantum Computer? MIT Technology Review. Retrieved December 7,2019 from https://www.technologyreview.com/s/612844/what-is-quantum-computing/

[7] PMI,2018. Success in Disruptive Times: Expanding the Value Delivery Landscape to Address the High Cost of Low Performance. Project Management Institute. Retrieved January 25, 2020 from https://www.pmi.org/-/media/pmi/documents/public/pdf/learning/thought-leadership/pulse/pulse-of-theprofession-2018.pdf

[8]Barima, O. K. B.,2010. Examination of the best, analogous, competing terms to describe value in construction projects. International Journal of Project Management, 28(3), 195-200.

[9] Oxford Learner's Dictionaries,2020. Quantum. Oxford Learner's Dictionaries Online. Oxford University Press. Retrieved October 25, 2019 from https://www.oxfordlearnersdictionaries.com /definition/ english/quantum 
[10] Cambridge Dictionary,2020. Quantum. Cambridge University Press. Retrieved October 25, 2019 from https://dictionary.cambridge.org/dictionary/english/quantum

[11] Coolman, R., 2014. What Is Quantum Mechanics? Live Science: September 26, 2014. Retrieved October 25, 2019 from https://www.livescience.com/33816-quantum-mechanics-explanation.html

[12] NASEM, 2019. Quantum Computing: Progress and Prospects. The National Academies of Sciences, Engineering, and Medicine. Washington, DC: The National Academies Press.

[13] Sarma,S.D., Freedman,M., and Nayak, C.,2006. Topological quantum computation. Physics Today 59(7), 32. Retrieved December 22, 2019 from https://doi.org/10.1063/1.2337825

[14] Orzel, C.,2015. What has Quantum Mechanics Ever done for Us? Forbes: August 13,2015.

[15]Gibney, E., 2019. Google publishes landmark quantum supremacy claim. Nature 574, 461-462. Retrieved December 29, 2019 from https://www.nature.com/magazine-assets/d41586-019-03213-z/d41586-019-03213z.pdf

[16]Ivakhnenko, O. V., Shevchenko, S. N., and Nori, F., 2018. Simulating quantum dynamical phenomena using classical oscillators: Landau-Zener-Stückelberg-Majorana interferometry, latching modulation, and motional averaging. Scientific Reports-Nature Research(Sci Rep) 8: 12218. Retrieved January 25, 2020 from doi: $10.1038 / \mathrm{s} 41598-018-28993-8$

[17] Greenemeier,L.,2018. How Close Are We-Really—to Building a Quantum Computer? Scientific American: May 30, 2018. Retrieved December 22, 2019 from https://www.scientificamerican.com/ article/how-close-are-we-really-to-building-a-quantum-computer/

[18] Nield, D. 2019. Physicists Just Achieved The First-Ever Quantum Teleportation Between Computer Chips. Science Alert December 31,2019. Retrieved January 25, 2020 from https://www.sciencealert.com/ scientists-manage-quantum-teleportation-between-computer-chips-for-the-first-time

[19] Pirandola, S., Eisert, J., Weedbrook, C., Furusawa, A., and Braunstein, S.L., 2015. Advances in quantum teleportation. Nature Photon 9, 641-652 (2015).

[20] Bennett, C. H., Brassard, G., Crepeau, C., Jozsa, R., Peres, A. \& Wootters, W. K. , 1993.Teleporting an unknown quantum state via dual classical and Einstein-Podolsky-Rosen channels. Physics Review Letters, 70(13), 1895-1899 (1993).

[21] Stephens, M., 2019. Synopsis: Quantum Teleportation Now Comes in 3D. Phys.org. Retrieved January 25, 2020 from https://physics.aps.org/synopsis-for/10.1103/PhysRevLett.123.070505

[22] Llewellyn, D., Ding, Y., Faruque, I.I., Paesani, S., Bacco, D., Santagati, R., Qian, Y., Li, Y., Xiao, Y., Huber, M., Malik, M., Sinclair, G. F., Zhou, X., Rottwitt, K., O’Brien, J.L, Rarity, J.G., Gong, Q., Oxenlowe, L.K., Wang,J. \& Thompson, M.G., 2019. Chip-to-chip quantum teleportation and multi-photon entanglement in silicon. Nature Physics(Nat. Phys.) 16,148-153. Retrieved January 25, 2020 from doi:10.1038/s41567-019-0727-x

[23] Luo, Y., Zhong,H., Erhard,M., Wang,X., Peng,L., Krenn, M., Jiang,X., Li, L., Liu,N., Lu,C., Zeilinger,A., and Pan,J., 2019.Quantum Teleportation in High Dimensions. Physics Review Letters, 123, 070505 (2019). 
[24] University of Vienna, 2019. Complex quantum teleportation achieved for the first time. Phys.org, News: August 23, 2019. Retrieved January 27, 2020 from https://phys.org/news/2019-08-complex-quantumteleportation.html

[25]Arute ,F., Arya, K., Babbush, R. , Bacon, D., Bardin, J. C., Barends, R., Biswas, R., Boixo, S., Brandao, F.G. S. L., Buell, D. A., Burkett, B., Chen, Y., Chen, Z., Chiaro, B., Collins, R., Courtney, W., Dunsworth, A., Farhi, E., Foxen, B., ...Martinis, J. M., 2019. Quantum supremacy using a programmable superconducting processor. Nature 574, 505-510. Retrieved January 25, 2020 from doi:10.1038/s41586-019-1666-5

[26]Gonzalez-Zalba, F., Yang,T., and Rossi,A.,2019. Manufacturing silicon qubits at scale. Physics World: 12, Nov. 2019.Retrieved January 27, 2020 from https://physicsworld.com/a/manufacturing-silicon-qubits-at-scale/

[27] He, Y., Gorman, S. K., Keith, D., Kranz, L., Keizer, J. G., \& Simmons. M. Y., 2019.A two-qubit gate between phosphorus donor electrons in silicon. Nature, 571, 371-375.

[28] Gibney, E., and Castelvecchi, D., 2016. Physics of 2D exotic matter wins Nobel. Nature News: October 4, 2016. Retrieved July 25, 2019 from https://www.nature.com/news/physics-of-2d-exotic-matter-wins-nobel-1.20722

[29]Gibney,E.,2016. Inside Microsoft's quest for a topological quantum computer. Nature News: October 21,2016. Retrieved December 22, 2019 from https://www.nature.com/articles/nature.2016.20774

[30] Savage,N.,2018. Building Quantum Computers With Photons: Silicon chip creates two-qubit processor. IEEE Spectrum: September 5, 2018. Retrieved December 23, 2019 from https://spectrum.ieee.org/techtalk/computing/hardware/building-quantum-computers-with-photons

[31] Preskill,J.,2018. Quantum Computing in the NISQ era and beyond. Quantum 2, 79(2018). Retrieved December 23, 2019 from https://doi.org/10.22331/q-2018-08-06-79

[32]Barima, O. K. B., 2008. Virtual concept use in the construction industry. In Becker, A. (Ed.), Electronic commerce: concepts, methodologies, tools, and applications, (chapter 65, pp. 799-806). Hershey, PA: IGI Global.

[33] Barima, O.K.B., 2009. Crucial tactical variables for value delivery in virtual projects. Automation in Construction, 18(7), 875-880. Retrieved August 2, 2020 from https://doi.org/10.1016/ j.autcon. 2009.03.014

[34] Li, J., Greenwood, D., and Kassem, M.,2019. Blockchain in the built environment and construction industry: A systematic review, conceptual models and practical use cases. Automation in Construction, 102, 288-307. Retrieved August 2, 2020 from https://doi.org/10.1016/j.autcon.2019.02.005

[35] LaRose,R.,2019. Overview and Comparison of Gate Level Quantum Software Platforms. Quantum,3,130 Retrieved November 7, 2020 from https://doi.org/10.22331/q-2019-03-25-130. https://quantum-journal.org/ instructions/authors/

[36] Quantiki,2020 .List of QC simulators. Retrieved November 7, 2020 from https://www. quantiki. org /wiki/list-qc-simulators

[37] Eisert, J., Hangleiter, D., Walk, N., Roth,I., Markham, D., Parekh, R., Chabaud, U \& Kashefi, E.2020. Quantum certification and benchmarking. Nature Reviews Physics, 2, 382-390(2020).

Retrieved November 7, 2020 from https://www.nature.com/articles/s42254-020-0186-4 
[38] 1Qbit,2019; Building the foundation for a quantum computing future. Retrieved January 28, 2020 from https://1qbit.com/

[39] IONQ,2019. A true Quantum leap. Retrieved January 28, 2020 from https://ionq.com/

[40] QbitLogic,2019. Building a New generation of Intelligent Systems for Tomorrow's Economies. Retrieved January 28, 2020 from http://www.qbitlogic.com/ 